\title{
Radial density profiles of PNe halos from numerical models of mass-loss history
}

\author{
Juan-Luis Verbena, Klaus-Peter Schröder and Astrid Wachter \\ Departamento de Astronomía, Universidad de Guanajuato \\ Apartado postal 144, 36000 Guanajuato, GTO, México \\ email: jluis@astro.ugto.mx
}

\begin{abstract}
We review the stellar mass loss of red giants and tip-AGB objects analizing the variation in the outflow velocity for different mass models (Wachter et al. 2002). We approach the superwind problem and see the evolution of tip-AGB stars via previously made mass-loss histories that are consistent with the Weidemann initial-final mass relationship (for carbon-rich stars). Finally density profiles are produced from these mass-loss histories, and the corresponding line-of-sight integration is compared with observational data (Phillips et al. 2009). We note the resemblance between the results obtained with our models and the observational data. We are thus able to reproduce the general trends of the emission from simple models (see Verbena et al. 2011).
\end{abstract}

Keywords. stars: AGB and post AGB, stars: winds outflows, planetary nebulae: general, circumstellar matter

\section{Introduction}

The density profiles of cool envelopes of young Planetary Nebulae (PNe) are reminiscent of the final AGB outflow history of the central star, so far as these have not yet been transformed by the hot wind and radiation of the central star. The evolution of the mass loss rate of that dust-driven, cool wind of the former giant in its final AGB stages must have shaped these envelopes to some extent. Less clear is the impact of changes in the outflow velocity.

A previous attemp with simpler physics - prescribed mass loss rates, no nucleation (Steffen et al. 1998) provided a similar result.

We here want to take a new and different look at this problem by using the behaviour of the underlying hydrodynamical wind models with respect to the outflow velocity. We are checking the assumption of constant outflow velocity in our work by checking the average velocities of the dust-driven wind models against dependence on the basic stellar quantities.

\section{Variation of the outflow velocity compared to the mass-loss history}

Figure 1 shows a graph of the outflow velocity besides the mass-loss history. For this investigation we use a set of hydrodynamical wind models that include formation and growth of dust grains which, by radiation pressure, drive massive outflows (Fleischer et al. 1992). These are characteristic of the ultimate stages of stellar evolution on the AGB. See also (Schröder et al. 1999)

As an example of mass-loss history, Fig. 1 shows the last 80000 years of mass-loss history of a $2.25 M_{\odot}$ star with solar element composition where we can see the superwind phase on the tip of the AGB with a duration of $\sim 20000$ years. 

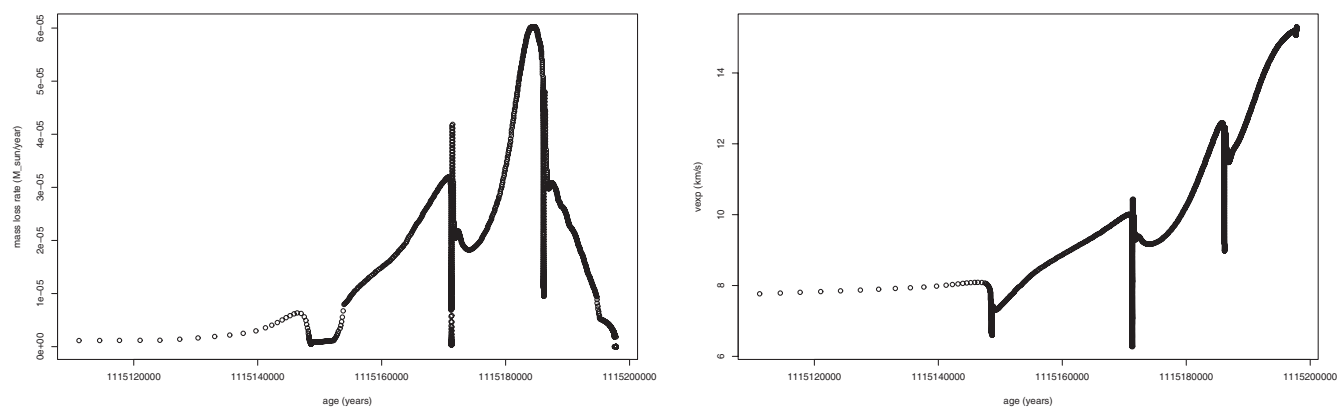

Figure 1. Outflow velocity (left) and mass-loss history (right) for a $2.25 \mathrm{M}_{\odot}$ star.
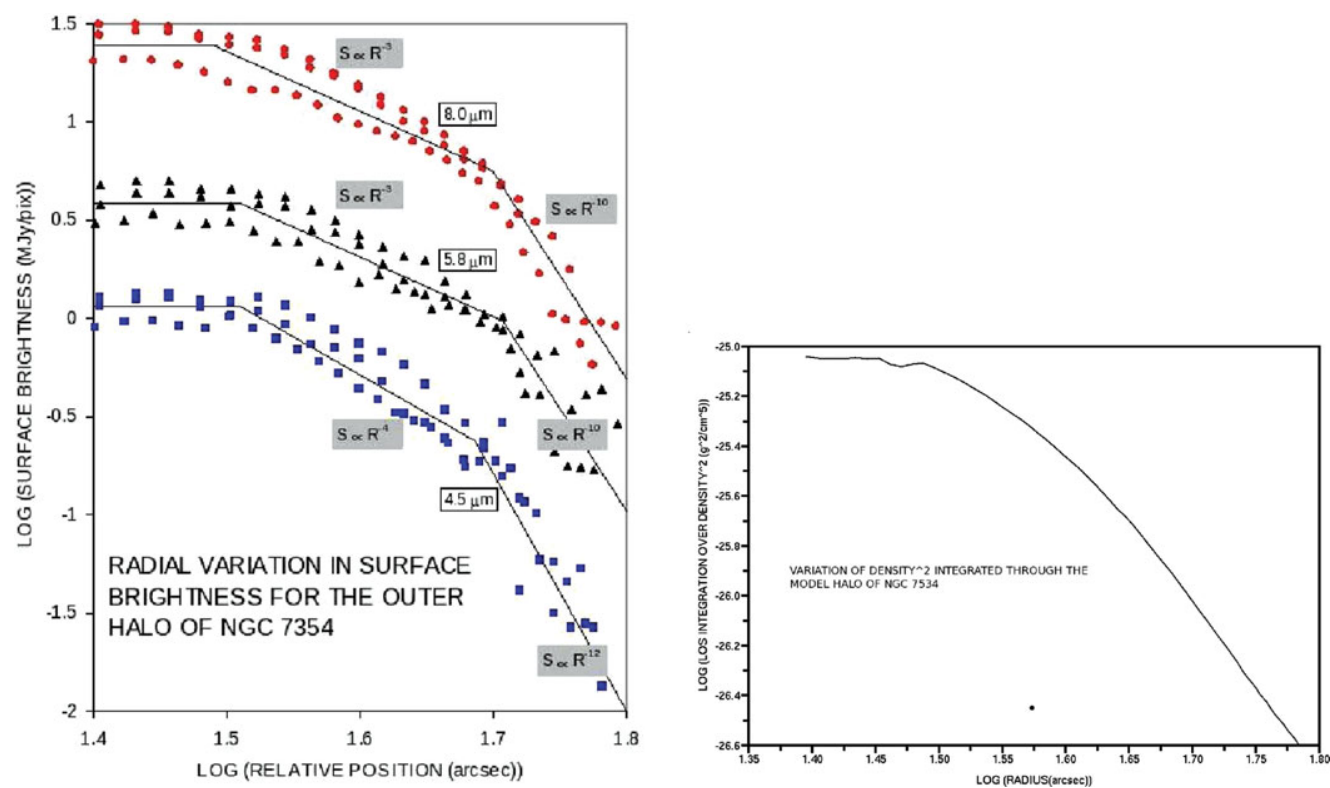

Figure 2. Observations in the mid-infrared (left) compared to line-of-sight integration of density squared (right).

\section{Density profiles}

Figure 2 shows the density profile and the integration of the density along the line of sight for comparison with observations (Phillips et al. 2009). Dilution in time is accounted for by the equation of continuity with constant outflow velocity.

\section{References}

Fleischer A. J., Gauger A., \& Sedlmayr E. 1992, A\& A, 266, 321

Phillips J. P., Ramos-Larios G., Schröder K. P., \& Verbena J. L. 2009, MNRAS, 399,1126

Schröder K. P., Winters J. M., \& Sedlmayr E. 1999, A\&A, 349, 898

Steffen M., Szczerba R., \& Schönberner D. 1998, A\&\&A, 337, 149

Verbena J. L., Schröder K. P., \& Wachter A. 2011, A\& A, 415, 2270

Wachter A., Schröder K. P., Winters J. M., Arndt T. U., \& Sedlmayr E. 2002, A\&AA, 384, 452 